\title{
PARITY VIOLATIONS IN HYDROGEN AND THE FUNDAMENTAL STRUCTURE OF THE WEAK CURRENT *
}

\author{
Robert N. CAHN and Gordon L. KANE \\ Physics Department, University of Michigan, Ann Arbor, MI 48109, USA
}

Received 13 January 1977

Revised manuscript received 15 June 1977

\begin{abstract}
Experiments in progress with hydrogen and deuterium may in practice determine the fundamental parameters and constituents of the weak interaction within the framework of unified gauge theories. In particular, for SU (2) $X$ $U(1)$ theories, from the results of these experiments one can infer the masses of the charged and neutral weak vector bosons (and thus $\sin ^{2} \theta \mathrm{W}$ ), and the isospin classification of the right-handed portions of the electron and the $u$ - and d-quarks. Non-singlet assignments for $e_{R}, u_{R}$ and $d_{R}$ would imply the existence of additional leptons and/or quarks. Hydrogen/deuterium data also may be used to discriminate between $\mathrm{SU}(2) \times \mathrm{U}(1)$ models and various models based on larger gauge groups.
\end{abstract}

Searches for parity violations in heavy atoms have shown that such effects, if present at all, are much smaller than predicted by the standard gauge theory of Weinberg and Salam [1]. Experiments with hydrogen should provide much more detailed information on the nature of weak interactions, as we shall see below. The idea of looking for parity violations due to neutral weak currents in hydrogen was suggested by Zel'dovich [2] in 1959 and explored further by Michel [3] in 1964. The importance of such experiments was increased, of course, by the discovery of neutral currents in neutrino interactions. More detailed discussions of parity violation experiments with hydrogen were given in 1975 by Azimov et al. [4], and by Lewis and Williams [5], who have been carrying out such experiments for some time. Closely related studies have been given by Feinberg and Chen [6].

Experiments with heavy atoms were undertaken first since the coherent effect of the full nucleus enhances the size of the parity violating effects over that in hydrogen. The interpretation of these experiments is obscured by the difficulty of estimating the wave functions in such complex systems. The hydrogenic system is ideal for a theoretical treatment, but much more difficult experimentally. However, Lewis and Williams pointed out [5] that the effect in hydrogen can be enhanced by applying a static magnetic field

\footnotetext{
^ Research supported in part by U.S.E.R.D.A.
}

to cross the energy levels which the parity violating interaction mixes, thus greatly increasing the degree of mixing.

The neutral weak current induces an effective Hamiltonian [7]

$H_{\mathrm{PV}}=-\frac{G}{\sqrt{2}}\left[C_{1 \mathrm{p}} \overline{\mathrm{e}} \gamma_{\mu} \gamma_{5} \mathrm{e} \overline{\mathrm{p}} \gamma^{\mu} \mathrm{p}+C_{2 \mathrm{p}} \overline{\mathrm{e}} \gamma_{\mu} \mathrm{e} \overline{\mathrm{p}} \gamma^{\mu} \gamma_{5} \mathrm{p}+\mathrm{p} \rightarrow \mathrm{n}\right]$.

A non-relativistic reduction shows that the $C_{1}$ 's arise from terms which are proportional to the nucleon density, while the $C_{2}$ 's depend on the nuclear spin. In heavy atoms, we expect the $C_{1}$ contribution to grow as $A$ or $Z$ relative to the $C_{2}$ contribution which never gets very large. The parity violation effect in heavy atoms thus is proportional to $\left(Z C_{1 \mathrm{p}}{ }^{+}\right.$ $\left.(A-Z) C_{1 \mathrm{n}}\right)$ times certain wave functions evaluated at the origin.

In contrast, the hydrogen/deuterium experiments can measure separately all four $C$ 's. There are three crossings in hydrogen and five in deuterium where the parity violating Hamiltonian causes mixing of s-states and p-states. It is these matrix elements which are determined experimentally by measuring the effect on transition rates of terms which are forbidden in the absence of parity violation [5]. In table 1 we give the matrix elements appropriate to the hydrogen and deuterium experiments.

From any gauge theory of weak interactions we can derive very simply the appropriate effective neutral 
Table 1

Matrix elements of the weak Hamiltonian, eq. (1) between $2 \mathrm{~S}_{1 / 2}$ and $2 \mathrm{P}_{1 / 2}$ states in hydrogen and deuterium. The energy unit is $\Delta=\sqrt{6} G_{\mathrm{F}} m_{\mathrm{e}^{\alpha^{4}} / 64 \pi} \approx 0.013 \mathrm{~Hz}$. The calculation is done for the high B-field limit so $M_{\mathrm{J}}$ is a good quantum number. See ref. [5]. The states have conventional normalization.

Hydrogen:

$\left\langle\mathrm{f}, M_{\mathrm{F}}=0\left|H_{\mathrm{PV}}\right| \beta, \quad M_{\mathrm{F}}=0\right\rangle$

$\left\langle\mathrm{f}, M_{\mathrm{F}}=-1\left|H_{\mathrm{PV}}\right| \beta, M_{\mathrm{F}}=-1\right\rangle$

〈e, $\left.M_{\mathrm{F}}=0\left|H_{\mathrm{PV}}\right| \beta, \quad M_{\mathrm{F}}=0\right\rangle$

Deuterium:

$$
\begin{aligned}
& \left\langle\mathrm{e}, M_{\mathrm{F}}=1 / 2\left|H_{\mathrm{PV}}\right| \beta, \quad M_{\mathrm{F}}=1 / 2\right\rangle=+\mathrm{i} \sqrt{2}\left(C_{2 \mathrm{p}}+C_{2 \mathrm{n}}\right) \times \Delta \\
& \left\langle\mathrm{e}, M_{\mathrm{F}}=-1 / 2\left|H_{\mathrm{PV}}\right| \beta, M_{\mathrm{F}}=-1 / 2\right\rangle=+\mathrm{i} \sqrt{2}\left(C_{2 \mathrm{p}}+C_{2 \mathrm{n}}\right) \times \Delta \\
& \text { (f, } \left.M_{\mathrm{F}}=1 / 2\left|H_{\mathrm{pV}}\right| \beta, \quad M_{\mathrm{F}}=1 / 2\right\rangle=-\mathrm{i}\left(C_{1 \mathrm{p}}+C_{1 \mathrm{n}}+C_{2 \mathrm{p}}+C_{2 \mathrm{n}}\right) \times \Delta \\
& \left\langle\mathrm{f}, M_{\mathrm{F}}=-1 / 2\left|H_{\mathrm{pV}}\right| \beta, M_{\mathrm{F}}=-1 / 2\right\rangle=-\mathrm{i}\left(C_{1 \mathrm{p}}+C_{1 \mathrm{n}}\right) \times \Delta \\
& \left\langle\mathrm{f}, M_{\mathrm{F}}=-3 / 2\left|H_{\mathrm{PV}}\right| \beta, M_{\mathrm{F}}=-3 / 2\right\rangle=-\mathrm{i}\left(+C_{1 \mathrm{p}}+C_{1 \mathrm{n}}-C_{2 \mathrm{p}}-C_{2 \mathrm{n}}\right) \times \Delta
\end{aligned}
$$

current Hamiltonian. The photon field, $A_{\mu}$, couples to fermions with a vertex -ie $\gamma^{\mu} Q$ where $Q$ is the charge operator. The $i$ th neutral weak gauge meson will couple with $-\mathrm{ie} \gamma^{\mu} O_{i}$ where $O_{i}$ is an operator which typically contains $\gamma_{5}$. The effective Hamiltonian between one fermion species $f$, and another, $f^{\prime}$ is

$H_{\mathrm{PV}}=\frac{e^{2}}{M_{\mathrm{W}}^{2}}\left(\overline{\mathrm{f}} \gamma_{\mu} O_{i} \mathrm{f}\right)\left(\overline{\mathrm{f}}^{\prime} \gamma^{\mu} O_{i} \mathrm{f}^{\prime}\right)$

We are interested in the instance when one fermion is an electron and the other fermion ranges over the quarks and anti-quarks in a nucleon. The operator $O_{i}$ can be separated into two parts: $O_{i}=O_{i}^{\mathrm{V}}+O_{i}^{\mathrm{A}} \gamma_{5}$. In order to determine the $C_{1}$ 's and $C_{2}$ 's we shall need the vector and axial vector charges associated with the various quarks,

〈proton, $\mathrm{p}, \mathrm{s}^{\prime}\left|\overline{\mathrm{q}}(0) \gamma_{\mu} \mathrm{q}(0)\right|$ proton, $\mathrm{p}, \mathrm{s}$ 〉

$$
=\overline{\mathrm{u}}\left(\mathrm{p}, \mathrm{s}^{\prime}\right) \gamma_{\mu} \mathrm{u}(\mathrm{p}, \mathrm{s}) N_{\mathbf{q}}^{\mathrm{V}},
$$

$\left\langle\right.$ proton, $\mathrm{p}, \mathrm{s}^{\prime}\left|\overline{\mathrm{q}}(0) \gamma_{\mu} \gamma_{5} \mathrm{q}(0)\right|$ proton, $\left.\mathrm{p}, \mathrm{s}\right\rangle$

$$
=\overline{\mathrm{u}}\left(\mathrm{p}, \mathrm{s}^{\prime}\right) \gamma_{\mu} \gamma_{5} \mathrm{u}(\mathrm{p}, \mathrm{s}) N_{\mathrm{q}}^{\mathrm{A}} \text {. }
$$

It is easy to see that the vector charges, $N_{\mathrm{q}}^{\mathrm{V}}$, are simply given by the naive quark content. The situation for the axial charge is slightly more complex. There are two isoscalar charges, one octet and one singlet, in addition to the isovector term. One simple way to express this is to consider formally the decays $n \rightarrow \mathrm{pe}^{-} \bar{\nu}_{\mathrm{e}}$ and $\Xi^{--} \rightarrow \Xi^{0} \mathrm{e}^{-} \bar{\nu}_{\mathrm{e}}[8]$. Assuming SU(3) we have

$F+D=N_{\mathrm{u}}^{\mathrm{A}}-N_{\mathrm{d}}^{\mathrm{A}}$,

$F-D=N_{\mathbf{d}}^{\mathbf{A}}-N_{\mathbf{s}}^{\mathbf{A}}$, where $F$ and $D$ are the usual coupling parameters with approximate values $F=0.425$ and $D=0.825\left(g_{\mathrm{A}}=\right.$ $F+D)$. Since the amount of strange and anti-strange quarks in a proton is believed to be very small and since in addition it is expected not to be very polarized, we ignore it henceforth (although it may be regarded as an additional, small, physical quantity which can be measured, in principle, in the hydrogen/deuterium experiments). To this very good approximation, we have $N_{\mathrm{u}}^{\mathrm{A}}=2 F, N_{\mathrm{d}}^{\mathrm{A}}=F-D$, as well as $N_{\mathrm{u}}^{\mathrm{V}}=2$, $N_{\mathrm{d}}^{\mathrm{V}}=1, N_{\mathrm{s}}^{\mathrm{V}}=0$. Quite generally, we can express the $C$ 's as

$$
\begin{aligned}
& C_{1 \mathrm{p}}=\sum_{i}\left(\frac{4 \pi \alpha \sqrt{2}}{G_{\mathrm{F}} M_{i}^{2}}\right) O_{i}^{\mathrm{A}}(\mathrm{e})\left[2 O_{i}^{\mathrm{V}}(\mathrm{u})+O_{i}^{\mathrm{V}}(\mathrm{d})\right] \\
& C_{1 \mathrm{n}}=\sum_{i}\left(\frac{4 \pi \alpha \sqrt{2}}{G_{\mathrm{F}} M_{i}^{2}}\right) O_{i}^{\mathrm{A}}(\mathrm{e})\left[O_{i}^{\mathrm{V}}(\mathrm{u})+2 O_{i}^{\mathrm{V}}(\mathrm{d})\right] \\
& C_{2 \mathrm{p}}=\sum_{i}\left(\frac{4 \pi \alpha \sqrt{2}}{G_{\mathrm{F}} M_{i}^{2}}\right) O_{i}^{\mathrm{V}}(\mathrm{e})\left[2 F O_{i}^{\mathrm{A}}(\mathrm{u})+(F-D) O_{i}^{\mathrm{A}}(\mathrm{d})\right] \\
& C_{2 \mathrm{n}}=\sum_{i}\left(\frac{4 \pi \alpha \sqrt{2}}{G_{\mathrm{F}} M_{i}^{2}}\right) O_{i}^{\mathrm{V}}(\mathrm{e})\left[(F-D) O_{i}^{\mathrm{A}}(\mathrm{u})+2 F O_{i}^{\mathrm{A}}(\mathrm{d})\right]
\end{aligned}
$$

where $M_{i}$ is the mass of the $i$ th neutral weak boson. These expressions completely determine all the effects of neutral current parity violation in any atom.

We consider the application of the general forms, eq. (5), first to $S U(2) \times U(1)$ theories. Here there is only one neutral weak boson, the $Z$. The operator $O$ for all the fermions is given by

$$
O=\left[\sin ^{2} \theta_{\mathrm{W}} Q-\frac{1}{2}\left(T_{3 \mathrm{~L}}+T_{3 \mathrm{R}}\right)-\frac{1}{2}\left(T_{3 \mathrm{R}}-T_{3 \mathrm{~L}}\right) \gamma_{5}\right],
$$


Table 2

Some typical examples of the parameters $C_{1 \mathrm{p}}, C_{1 \mathrm{n}}, C_{2 \mathrm{p}}, C_{2 \mathrm{n}}$ which can be measured in hydrogen/deuterium parity violation experiments. For each model, the parameters are determined by $K=M_{\mathrm{W}}^{2} \sec ^{2} \theta_{\mathrm{W}} / M_{\mathrm{Z}}^{2}, \sin ^{2} \theta \mathrm{W}$, and the axial vector coupling constants $F \approx 0.425, D \approx 0.825, g_{\mathrm{A}}=F+D$ (see eqs. (7) and (8)). The entries follow from eq. (9).

\begin{tabular}{|c|c|c|}
\hline & $T_{3 \mathrm{R}}^{\mathrm{e}}=0$ & $T_{3 \mathrm{R}}^{\mathrm{e}}=-1 / 2$ \\
\hline$T_{3 \mathrm{R}}^{\mathrm{u}}=0 \quad T_{3 \mathrm{R}}^{\mathrm{d}}=0$ & $\begin{array}{l}C_{1 \mathrm{p}}=K\left(1-4 \sin ^{2} \theta \mathrm{W}\right) / 2 \\
C_{1 \mathrm{n}}=-K / 2 \\
C_{2 \mathrm{p}}=K\left(1-4 \sin ^{2} \theta \mathrm{W}\right)(F+D) / 2 \\
C_{2 \mathrm{n}}=-K\left(1-4 \sin ^{2} \theta \mathrm{W}\right)(F+D) / 2\end{array}$ & $\begin{array}{l}C_{1 \mathrm{p}}=0 \\
C_{1 \mathrm{n}}=0 \\
C_{2 \mathrm{p}}=K\left(2-4 \sin ^{2} \theta \mathrm{W}\right)(F+D) / 2 \\
C_{2 \mathrm{n}}=-K\left(2-4 \sin ^{2} \theta \mathrm{W}\right)(F+D) / 2\end{array}$ \\
\hline$T_{3 \mathrm{R}}^{\mathrm{u}}=1 / 2 \quad T_{3 \mathrm{R}}^{\mathrm{d}}=0$ & $\begin{array}{l}C_{\mathrm{Ip}}=K\left(3-4 \sin ^{2} \theta_{\mathrm{W}}\right) / 2 \\
C_{\mathrm{In}}=0 \\
C_{2 \mathrm{p}}=K\left(1-4 \sin ^{2} \theta_{\mathrm{W}}\right)(D-F) / 2 \\
C_{2 \mathrm{n}}=-K\left(1-4 \sin ^{2} \theta_{\mathrm{W}}\right) F\end{array}$ & $\begin{array}{l}C_{1 \mathrm{p}}=0 \\
C_{1 \mathrm{n}}=0 \\
C_{2 \mathrm{p}}=K\left(2-4 \sin ^{2} \theta \mathrm{W}\right)(D-F) / 2 \\
C_{2 \mathrm{n}}=-K\left(2-4 \sin ^{2} \theta \mathrm{W}\right) F\end{array}$ \\
\hline$T_{3 \mathrm{R}}^{\mathrm{d}}=-1 / 2$ & $\begin{array}{l}C_{1 \mathrm{p}}=-2 K \sin ^{2} \theta_{\mathrm{W}} \\
C_{1 \mathrm{n}}=-3 K / 2 \\
C_{2 \mathrm{p}}=K\left(1-4 \sin ^{2} \theta_{\mathrm{W}}\right) F \\
C_{2 \mathrm{n}}=K\left(1-4 \sin ^{2} \theta_{\mathrm{W}}\right)(F-D) / 2\end{array}$ & $\begin{array}{l}C_{1 \mathrm{p}}=0 \\
C_{1 \mathrm{n}}=0 \\
C_{2 \mathrm{p}}=K\left(2-4 \sin ^{2} \theta_{\mathrm{W}}\right) F \\
C_{2 \mathrm{n}}=K\left(2-4 \sin ^{2} \theta_{\mathrm{W}}\right)(F-D) / 2\end{array}$ \\
\hline$T_{3 \mathrm{R}}^{\mathrm{u}}=1 / 2 \quad T_{3 \mathrm{R}}^{\mathrm{d}}=-1 / 2$ & $\begin{array}{l}C_{1 \mathrm{p}}=K\left(2-4 \sin ^{2} \theta \mathrm{W}\right) / 2 \\
C_{1 \mathrm{n}}=-K \\
C_{2 \mathrm{p}}=0 \\
C_{2 \mathrm{n}}=0\end{array}$ & $\begin{array}{l}C_{1 \mathrm{p}}=0 \\
C_{1 \mathrm{n}}=0 \\
C_{2 \mathrm{p}}=0 \\
C_{2 \mathrm{n}}=0\end{array}$ \\
\hline
\end{tabular}

where $T_{3}$ is the third generator of the weak SU(2). (The matrix elements of (6) and similar forms below contribute to eq. (5), with unity or $\gamma_{5}$ giving coefficients $N_{\mathrm{u}, \mathrm{d}}^{\mathrm{A}, \mathrm{V}}$ via eqs. (3) and (4)). The various fermions may be assigned different values of $T_{3}$ for their right handed $\left(T_{3 \mathrm{R}}\right)$ and left handed components $\left(T_{3 \mathrm{~L}}\right)$. In the simplest theory, that of Weinberg [9] and Salam [10], and Glashow et al. [11], all right handed fermions are SU(2) singlets, and all left handed fermions are doublets. The next simplest theories are obtained by adding some right handed doublets. For example, if the electron appears as the lower component of a right handed doublet (with a new neutral lepton above it), $T_{3 \mathrm{R}}^{\mathrm{e}}=-\frac{1}{2}$. It is also possible to introduce right handed partners for the u-quarks (usually designated $b$ ) and for the d-quark (usually designated $t$ ). Thus there are eight simple variations of $S U(2) \times U(1)$, and these are shown in table 2 . We have used the notation

$K=\frac{M_{\mathrm{W}}^{2}}{\cos ^{2} \theta M_{\mathrm{Z}}^{2}}=\frac{4 \pi \alpha \sqrt{2}}{8 G M_{\mathrm{Z}}^{2} \sin ^{2} \theta_{\mathrm{W}} \cos ^{2} \theta_{\mathrm{W}}}$.

The value of $K$ depends on the Higgs bosons of the theory. In the simplest theories it is unity, but in general must be considered an unknown parameter.

In most theories the values of $T_{3}$ are integers or half integers, al though more complex schemes can exist (e.g. with flavor changing neutral currents), in which the quarks are not eigenstates of $T_{3}$ and mixing angles appear. If the former is the case, it would be relatively easy to distinguish between the eight cases shown in table 2 if experiments were performed giving the $C$ 's to a precision \pm 0.1 . If the more complex schemes are considered, either with mixing (not just Cabibbo type mixing which leave $T_{3 L, \mathrm{R}}$ as an integer or half integer) or with larger gauge groups it is not possible to say ahead of time which parameters will be determined by the hydrogen/deuterium experiment. However, the qualitative point is simple: the hydrogen/deuterium experiment since it can measure four separate aspects of the neutral weak interaction will be one of the most decisive experiments for weak current theory.

We consider next some theories with gauge groups other than $\mathrm{SU}(2) \times \mathrm{U}(1)$. One of the most striking is that of Mohapatra and co-workers [12]. Their SU(2) $\times \mathrm{SU}(2)_{\mathrm{R}} \times \mathrm{U}(1)$ theory has two neutral vector mesons, one of which couples only with a vector coupling, and the other of which couples only axially. Clearly, then, there are no parity violations expected with such a model in either heavy atoms or hydrogen. The apparent parity violation suggested by the difference in neutrino and anti-neutrino neutral current cross sections is a consequence of the "polarized" neu- 
Table 3

Typical values for the measurable parameters $C_{1 \mathrm{p}}, C_{1 \mathrm{n}}, C_{2 \mathrm{p}}$, $C_{2 n}$. For the purposes of comparison we have chosen $\sin ^{2} \theta \mathrm{W}$ $=1 / 3, K=1$ for $\operatorname{SU}(2) \times U(1)$ models, $\cos ^{2} \beta=1 / 2$ for the model of ref. [13], and $\sin ^{2} \theta_{3}=0.2, K^{\prime}=1$ for the model of ref. [15].

\begin{tabular}{lcccc}
\hline & $C_{1 \mathrm{p}}$ & $C_{1 \mathrm{n}}$ & $C_{2 \mathrm{p}}$ & $C_{2 \mathrm{n}}$ \\
\hline WSGIM & -0.17 & -0.5 & -0.21 & +0.21 \\
$b$ & +0.83 & 0 & -0.07 & +0.14 \\
$t$ & -0.67 & -1.5 & -0.14 & +0.07 \\
$b, t$ & +0.33 & -1 & 0 & 0 \\
$E^{0}$ & 0 & 0 & +0.42 & -0.42 \\
$E^{0}, b$ & 0 & 0 & 0.13 & -0.28 \\
$E^{0}, t$ & 0 & 0 & +0.28 & -0.13 \\
$E^{0}, b, t$ & 0 & 0 & 0 & 0 \\
Mohapatra-Sidhu & 0 & 0 & 0 & 0 \\
SU(2) $\times$ SU(2) $\times \mathrm{U}(1)$ & 0 & & & \\
DGG & & & & \\
SU(2) $\times$ SU(2) $\times \mathrm{U}(1)$ & -0.08 & -0.25 & -0.10 & +0.10 \\
Lee-Weinberg & & & & \\
SU(3) $\times \mathrm{U}(1)$ & 0 & 0 & 0.22 & -0.10 \\
\hline.
\end{tabular}

trinos and the interference between the two neutral vector exchanges.

Another $\mathrm{SU}(2)_{\mathrm{L}} \times \mathrm{SU}(2)_{\mathrm{R}} \times \mathrm{U}(1)$ theory has been proposed by De Rujula et al. [13]. Of the two neutral weak vector mesons in this theory, one is pure vector and the other is identical to the $\mathrm{Z}$ in the simple Weinberg theory. In the effective Lagrangian, the parity violating term appears multiplied by a coefficient, $\cos ^{2} \beta$. This parameter is determined by fitting data in other experiments and is found to be about $0.4-0.5$. Thus the predictions for parity violation in atoms are just the same as the simple Weinberg theory, except for an overall factor of $0.4-0.5$.

The gauge group SU(3) $\times U(1)$ was proposed earlier by Segre and Weyers [15] and variations have been considered by Langacker and Segre [16] and by Segre and Golshani [17]. Some versions have purely vector couplings to the electron (as in the LeeWeinberg model discussed below) while others have substantial axial electron couplings and thus predict sizeable effects in heavy atom experiments. The SU(3) $X \mathrm{U}(1)$ model, of Lee and Weinberg [14] has quite different physical consequences. There are three diagonal neutral currents: in contrast to the Segre-Weyers model, both of the diagonal neutral weak currents are associated with mesons of moderate mass. The relevant fermion multiplets are $\left(\mathrm{u}^{\prime}, \mathrm{d}, \mathrm{b}^{\prime}\right)_{\mathrm{L}} ;\left(\mathrm{t}^{\prime}, \mathrm{b}^{\prime}, \mathrm{d}\right)_{\mathrm{R}}$; $\left(\nu_{\mathrm{e}}, \mathrm{e}^{-}, \mathrm{E}^{-\prime}\right)_{\mathrm{L}}$; and $\left(\mathrm{E}^{0 \prime}, \mathrm{E}^{-\prime}, \mathrm{e}^{-}\right)_{\mathrm{R}}$. The quark triplets have $y=0$, where $y$ is the generator of the $U(1)$ symmetry. The leptons have $y=-2 / 3$. The charge is $Q=\lambda_{3} / 2+\lambda_{8} / 2 \sqrt{3}+y I$. Because of the discrete symmetries of this theory, $u_{R}$ must be an $\mathrm{SU}(3)$ singlet. Consequently, its value of $y$ is $2 / 3$. The couplings of the physical neutral weak vector mesons (which are diagonal) are found to be

$O_{Y}=\frac{1}{\sqrt{3} \sin \theta_{3}}\left[\frac{\lambda_{3}}{2}-\frac{\sqrt{3}}{2} \lambda_{8}\right]=\frac{1}{\sqrt{3} \sin \theta_{3}}\left(\begin{array}{rrr}0 & 0 & 0 \\ 0 & -1 & 0 \\ 0 & 0+1\end{array}\right)$,

$O_{Z}=\left[Q \cot \theta_{3}-y / \sin \theta_{3} \cos \theta_{3}\right]$,

where $\theta_{3}$ is a mixing angle analogous to $\theta_{\mathrm{W}}$, and the charged $W$ mass is given by $m^{2}(W)=4 \pi \alpha / 3 \sqrt{2} G_{\mathrm{F}} \sin ^{2} \theta_{3}$. As noted by Lee and Weinberg, this means that the $Y$ does not contribute to parity violation in atoms, and the coupling of the $Z$ to electrons is pure vector [15]. Thus the parity violations in heavy atoms should vanish in the approximation in which only the $C_{1}$ terms are retained. On the other hand, the axial coupling of the $\mathrm{Z}$ to $\mathrm{u}$-quarks is not zero since the left and right handed $u$-quarks have different values of $y$ : restricted to $u$ quarks, we have $y=\left(1+\gamma_{5}\right) / 3$. From eq. (5), we find

$C_{2 \mathrm{p}}=4 K^{\prime}\left(\cos ^{2} \theta_{3}-2 / 3\right) F$, $C_{2 \mathrm{n}}=4 K^{\prime}\left(\cos ^{2} \theta_{3}-2 / 3\right)(F-D) / 2$,

where $K^{\prime}=M_{\mathrm{W}}^{2} /\left(\cos ^{2} \theta_{3} M_{\mathrm{Z}}^{2}\right)$ is expected to be near unity. Note that the relative sign of $F$ and $F-D$ will be negative even if the detailed values are not accurate, so $C_{2 \mathrm{p}} / C_{2 \mathrm{n}}$ is negative.

In table 3 we have listed some nominal values for the $C$ 's in the theories discussed above. For the SU(2) $X \mathrm{U}(1)$ theories we have taken $K=1$ and $\sin ^{2} \theta_{\mathrm{W}}=1 / 3$. For the $S U(2) \times S U(2) \times U(1)$ model of De Rujula et al., we have taken $\sin ^{2} \theta_{W}=1 / 3, \cos ^{2} \beta=1 / 2$. For the SU(3) $\times \mathrm{U}(1)$ model of Lee and Weinberg we have taken $\sin ^{2} \theta_{3}=0.2, K^{\prime}=1$.

The data from experiments with bismuth conducted at the University of Washington and at Oxford University show that the parity violations are no larger than about $10 \%$ or so of the prediction of the standard WeinbergSalam model (the first entry of tables 2 and 3) [1]. While there have been some criticisms of the theoretical calculations of the atomic physics associated with these experiments, it appears unlikely that this can be the source of such a large discrepancy. The most attractive 
explanation is that there is a pure vector coupling of neutral currents to electrons. Table 3 contains several such theories (the ones with zero for the $C_{1}$ 's). The hydrogen/deuterium experiments should provide valuable information for discriminating between some of these theories. From the table it is apparent that experiments should aim for a precision of at least 0.1 for the values of the $C$ 's.

Many other experiments will provide important information about the nature of weak interactions. The most decisive information is likely to come from the experiments whose interpretation is the most direct. Certainly elastic neutrino-electron scattering is of central importance. Scattering of polarized electrons from nucleons is similar in many ways to the hydrogen/ deuterium experiments discussed above and may well provide important information. Neutrino scattering from nucleons will of course provide basic data, but it must be kept in mind that the thresholds for production of new flavors may be very high. If this is so, then the neutral current data may be the most significant.

The hydrogen/deuterium experiments have a unique place among these experiments. They offer the clarity of interpretation possessed by the elastic neutrinoelectron scattering while yielding information about the hadronic as well as leptonic facet of weak interactions. Increasingly precise measurements of the properties of the hydrogen atom have been a catalyst for the discovery of the fundamental laws of nature. The crude hydrogen spectrum led to Bohr's theory. The fine structure gave confirmation to Dirac's relativistic electron theory. The Lamb shift played a central role in the development of quantum electrodynamics.
It appears that hydrogen will now play a crucial role in elucidating the fundamental nature of both weak and strong interactions.

\section{References}

[1] P.E.G. Baird et al., Nature 264 (1976) 528; P. Sandars, W.L. Williams, invited talks at the Washington Meeting of the A.P.S., April 1977.

[2] Ya. Zel'dovich, JETP 9 (1959) 682.

[3] F.C. Michel, Phys. Rev. B138 (1965) 408.

[4] Ya. Azimov et al., JETP 40 (1975) 8.

[5] R.R. Lewis and W.L. Williams, Phys. Lett. 59B (1975) 70 see also R.W. Dunford, R.R. Lewis and W.L. Williams, Parity nonconservation in the hydrogen atom, II and III, Univ. Mich. preprints.

[6] G. Feinberg and M.Y. Chen, Phys. Rev. D10 (1974) 190; D10 (1974) 3145, and D10 (1974) 3789.

[7] We use the notation of ref. [6].

[8] L.M. Sehgal, Phys. Rev. D10 (1974) 1663.

[9] S. Weinberg, Phys. Rev. Lett. 19 (1967) 1264.

[10] A. Salam in Elementary particle physics, ed. N. Svarntholm (Almquist and Wiksells, Stockholm 1968) p. 367.

[11] S.L. Glashow, J. Iliopoulos, L. Maiani, Phys. Rev. D2 (1970) 1285 .

[12] R.N. Mohapatra and D.P. Sidhu, Phys. Rev. Lett. 38 (1977) 667.

[13] A. De Rujula, H. Georgi and S.L. Glashow, to be published.

[14] B.W. Lee and S. Weinberg, Phys. Rev. Lett. 38 (1977) 1237 ;

B.W. Lee, R. Slirock and S. Weinberg, to be published.

[15] G. Serge and J. Weyers, Phys. Lett. 65B (1976) 243.

[16] P. Langacker and G. Segre, Heavy leptons and trimuons in an SU(3) $\times$ U (1) model, UPR-0073T (April 1977) unpublished.

[17] G. Segre and M. Golshani, Univ. of Penn. preprint UPR0075T, unpublished. 